\title{
Reversible Switching of the Dirac Point in Graphene Field-Effect Transistors Functionalized with Responsive Polymer Brushes
}

\author{
Esteban Piccinini, ${ }^{\dagger}$ Christina Bliem, ${ }^{\dagger}$ Juan M. Giussi, ${ }^{\dagger}$ Wolfgang Knoll, ${ }^{\dagger}, \S$ and Omar Azzaroni ${ }^{*}, \dagger, \|_{\odot}$ \\ ${ }^{\dagger}$ Departamento de Química, Facultad de Ciencias Exactas, Instituto de Investigaciones Fisicoquímicas Teóricas y Aplicadas \\ (INIFTA), Universidad Nacional de La Plata (UNLP), CONICET, Suc. 4-C.C.16, 1900 La Plata, Argentina \\ ${ }^{\ddagger}$ Biosensor Technologies and ${ }^{\S}$ Competence Center for Electrochemical Surface Technology (CEST), Austrian Institute of \\ Technology (AIT), Konrad-Lorenz-Strasse 24, 3430 Tulln, Austria \\ "CEST-UNLP Partner Lab for Bioelectronics, Diagonal 64 y 113, La Plata 1900, Argentina
}

Supporting Information

ABSTRACT: The reversible control of the graphene Dirac point using external chemical stimuli is of major interest in the development of advanced electronic devices such as sensors and smart logic gates. Here, we report the coupling of chemoresponsive polymer brushes to reduced graphene oxide (rGO)-based field-effect transistors to modulate the graphene Dirac point in the presence of specific divalent cations. Poly[2-(methacryloyloxy)ethyl] phosphate (PMEP) brushes were grown on the transistor channel by atom transfer radical polymerization initiated from amine-pyrene linkers noncovalently attached to rGO surfaces. Our results show an increase in the Dirac point voltage due to electrostatic gating effects upon the specific binding of $\mathrm{Ca}^{2+}$ and $\mathrm{Mg}^{2+}$ to the PMEP brushes. We demonstrate that the electrostatic gating is reversibly controlled by the charge density of the polymer brushes, which depends on the divalent cation concentration. Moreover, a theoretical formalism based on the Grahame equation and a Langmuir-type binding isotherm is presented to obtain the PMEP-cation association constant from the experimental data.

\section{INTRODUCTION}

Graphene, a two-dimensional semiconducting material, is one of the most promising materials for post-silicon electronics due to its outstanding physical and electronic properties. ${ }^{1-3}$ Due to its great sensitivity, graphene can be used as the chemo-toelectrical signal transducing material in field-effect transistors (FETs) for the generation of high-performance responsive devices, such as advanced sensors and smart logic gates. ${ }^{4-7}$ For these applications, the reversible modulation of the graphene electronic properties (i.e., the doping level) by a specific external (bio)chemical stimulus represents a key feature. ${ }^{8,9}$ The chemoelectrical signal transduction in graphene FETs is based on various mechanisms, including charge transfer, charge scattering, and capacitive and electrostatic field effects. ${ }^{10}$ Among them, the electrostatic field effect (also referred to as electrostatic gating) is widely regarded as the most reliable sensing mechanism ${ }^{11}$ and consists of the modulation of the Fermi level by means of an external electric field, for example, the local electric field induced by the adsorption of charged macromolecules. ${ }^{12,13}$ The modulation of the Fermi level by electrostatic gating is observed as a shift in the Dirac point voltage.

To confer specificity to the transducer, recognition elements need to be anchored on the graphene surface. For instance, the use of monoclonal antibodies or enzymes as recognition elements has led to FET sensors with low limits of detection and high sensitivity. ${ }^{14-17}$ Unfortunately, the biomolecule activity is affected by changes in temperature, $\mathrm{pH}$, ionic strength, and the presence of inhibitors. Furthermore, irreversible denaturation of the biomolecule structure with complete loss of its functional activity can occur in harsh conditions. ${ }^{18}$ This issue is an important limitation for the development of FET devices for sensing applications. ${ }^{19}$ To solve this problem, synthetic and robust "recognition elements" anchored on the transducer surface should be used.

Regarding the electrostatic gating sensing mechanism, the synthetic receptors have to display controllable charge density as a function of specific target concentration and be located directly onto graphene. In this sense, chemoresponsive polyelectrolytes seem to be an ideal "recognition element" because they display high charge density that is controllable via chemical means. ${ }^{20,21}$ Hence, the use of polymer brushes ${ }^{22,23}$ is an attractive alternative to generate synthetic and robust responsive interfaces ${ }^{24-31}$ for FET sensing devices. ${ }^{32-35}$ As an example, Hess et al. $^{35}$ reported the covalent functionalization of graphene with polymer brushes containing weak cation monomer units ( $N, N$-dimethylaminoethyl methacrylate, DMAEMA). Due to the polymer brush's nature, the $\mathrm{pH}$ sensing range of the graphene FETs has been modified, enhancing the enzymatic detection of acetylcholine. ${ }^{35}$ Since

Received: March 27, 2019

Revised: May 13, 2019

Published: May 16, 2019 
graphene FETs present the inherent property of sensing $\mathrm{pH}$ changes, ${ }^{36}$ the DMAEMA monomer units do not confer on the graphene FETs a new recognition function, but they act as an enhancer of the one already present. Therefore, the use of stimuli-responsive polyelectrolytes to confer on the graphene interface a new selective interaction is still a challenging task. In this regard, polymer brushes containing phosphate monomer units turn out to be a promising system to be studied because on the one hand, they present high charge density, and on the other hand, they can form strong complexes with some divalent cations (e.g., $\mathrm{Ca}^{2+}$ and $\left.\mathrm{Mg}^{2+}\right) .{ }^{37,38}$

In this work, we report the polymerization of poly[2(methacryloyloxy)ethyl] phosphate (PMEP) brushes on reduced graphene oxide ( $\mathrm{rGO}$ ) FETs to confer on them a cation selective response $\left(\mathrm{Ca}^{2+}\right.$ and $\left.\mathrm{Mg}^{2+}\right)$. The growth of responsive PMEP polymer brushes was accomplished via surface-initiated atom transfer radical polymerization (SIATRP), a powerful approach to tune/adapt the chemical and physical properties of surfaces. ${ }^{39-41}$ Surface modification with PMEP brushes was characterized by X-ray photoelectron spectroscopy (XPS), ellipsometry, atomic force microscopy (AFM), and contact angle goniometry. Then, the field-effect properties of the PMEP-modified graphene transistors, in particular the Dirac point voltage, were studied in the presence of different types of cations. Finally, a theoretical formalism was introduced to obtain the PMEP-cation association constant from the experimental values of the Dirac point voltage at different $\mathrm{Ca}^{2+}$ concentrations. Our results show a strong influence of the PMEP brushes on the rGO FET response provided that the presence of divalent ions $\mathrm{Ca}^{2+}$ and $\mathrm{Mg}^{2+}$ promotes significant changes in the Dirac point of graphene. We believe that these results further broaden the scope of action of polymer brushes to emerging technological areas, namely, graphene-based field-effect transistors.

\section{EXPERIMENTAL SECTION}

Materials. Interdigitated microelectrodes (ED-IDA1-Au) as well as an electrochemical batch cell were obtained from Micrux Technologies (Oviedo, Spain). A concentrated graphene oxide solution was kindly supplied by Prof. Josef Breu's laboratory (University of Bayreuth, Germany). Anhydrous $N, N$-dimethylformamide (99.8\%), hydrazine monohydrate (99\%), (3-aminopropyl)triethoxysilane (97\%), 1-amine pyrene (97\%), $\alpha$-bromoisobutyryl bromide (98\%), triethylamine (99\%), 2,2'-bipyridyl (99\%), $\mathrm{CuBr}_{2}$ (99.999\%), and 2-(methacryloyloxy)ethyl phosphate (referred to as MEP, 90\%) were purchased from Sigma-Aldrich. THF (PA) was purchased from Anedra S.A. This solvent was dried by refluxing in the presence of sodium (Aldrich, 99.8\%) and benzophenone (Aldrich, 99\%) until the observation of purple coloration and then distilled.

Graphene-Based Transistor Fabrication. The reduced graphene oxide field-effect transistors (rGO FETs) were prepared from interdigitated electrodes as we described in previous works. ${ }^{16,42}$

Poly[2-(methacryloyloxy)ethyl] phosphate (PMEP) Brush Growth on the Transistor Channel. PMEP brushes were obtained by surface-initiated atom transfer radical polymerization (SI-ATRP) on initiator-modified rGO-FETs. The functionalization involved three synthetic steps: (1) noncovalent functionalization of the graphene surface with amine-pyrene, (2) acylation of amine-pyrene groups with $\alpha$-bromoisobutyryl bromide to prepare the surface-confined ATRP initiator, and (3) ATRP of 2-(methacryloyloxy)ethyl phosphate (MEP).

1. rGO-FET modification with amine-pyrene: rGO-FET chips were incubated in $2 \mathrm{mM}$ amine-pyrene solution prepared in dimethylformamide (DMF) for $4 \mathrm{~h}$, rinsed with DMF and Milli- $\mathrm{Q}$ water, and finally dried with $\mathrm{N}_{2}$.
2. Acylation of amine-modified rGO-FETs with $\alpha$-bromoisobutyryl bromide to obtain ATRP initiator sites: ${ }^{43}$ amine-modified rGO-FET substrates were immersed in an Erlenmeyer containing $40 \mathrm{~mL}$ of dry THF and $2 \mathrm{~mL}$ of triethylamine under $\mathrm{N}_{2}$. After this, the reaction solution was placed in an ice bath, and $2 \mathrm{~mL}$ of $\alpha$-bromoisobutyryl bromide was added slowly using a pressure-equalizing dropping funnel. After $3 \mathrm{~h}$, the transistors were removed and washed with acetone, ethanol, and Milli-Q water.

3. Growth of PMEP brushes from immobilized initiators: ATRP was carried out according to the method described by Zhou et al. ${ }^{44}$ MEP (12.5 g) was dissolved in $20 \mathrm{~mL}$ of water and neutralized with $\mathrm{NaOH}$ solution to produce the disodium salt. Then, $0.4 \mathrm{~g}$ of $2,2^{\prime}$ bipyridyl and $0.011 \mathrm{~g}$ of $\mathrm{CuBr}_{2}$ were added, and the solution was degassed by $\mathrm{N}_{2}$ bubbling for $1 \mathrm{~h}$. Then, $0.144 \mathrm{~g}$ of $\mathrm{CuBr}$ was added, and the mixture was left for 15 min under $\mathrm{N}_{2}$. Simultaneously, ATRP initiator-modified rGO-FETs were sealed in Schlenk tubes and degassed through vacuum $/ \mathrm{N}_{2}$ cycles. Then, the reaction mixture was syringed into these Schlenk tubes, adding enough to cover the samples completely. The mixture was left overnight under $\mathrm{N}_{2}$ at 60 ${ }^{\circ} \mathrm{C}$, and then the substrates were removed, washed with deionized water, and dried with $\mathrm{N}_{2}$.

Field-Effect Transistor Characterization. Liquid-gated graphene FETs were measured using the batch cell from Micrux Technologies. Electrical measurements were performed by applying a $100 \mathrm{mV}$ source-drain bias and sweeping the gate potential $\left(V_{\mathrm{g}}\right)$ while monitoring the current between the drain and the source $\left(I_{\mathrm{ds}}\right)$ using a Teq4 bipotensiostat (nanoTeq, Buenos Aires, Argentina). A Ag/ $\mathrm{AgCl}$ reference electrode from BASi (Indiana, USA) was used as a gate electrode.

X-ray Photoelectron Spectroscopy. An SPECS SAGE HR 100 $\mathrm{X}$-ray photoelectron (XPS) spectrometer was used. A Mg K $\alpha$ (1253.6 $\mathrm{eV}) \mathrm{X}$-ray source was employed operating at $12.5 \mathrm{kV}$ and $10 \mathrm{~mA}$. Quantitative analysis of spectra was carried out by using the Casa XPS software, employing Shirley baselines and Gaussian\Lorentzian functions. Surface-charging effects were corrected by setting the binding energy of the main component of the C1s core level at 284.6 $\mathrm{eV}$.

Contact Angle Goniometry. A Ramé-Hart contact angle system (model 290 ) at $25^{\circ} \mathrm{C}$ was used using $1 \mu \mathrm{L}$ drops. The average contact angle value was obtained at three different locations of the same sample.

Atomic Force Microscopy (AFM). A Veeco Multimode AFM connected to a NanoScope $\mathrm{V}$ controller was used to image the polymer brushes. AFM measurements were performed in tapping mode with a sample scan size of $2.5 \mu \mathrm{m} \times 2.5 \mu \mathrm{m}$. Nonconductive sharpened silicon nitride probes (Bruker, $K=0.12 \mathrm{Nm}^{-1}$ ) were used.

Ellipsometry. For the PMEP film thickness estimation, an alphaSE ellipsometer from Woollam Co. Inc. was used. The ellipsometric measurements were conducted using a wavelength range from 550 to $900 \mathrm{~nm}$ and incidence angle of $70^{\circ}$ in high-precision mode. A multilayer model taking into account the layers of different materials (i.e., APTES, rGO, amine-pyrene, and PMEP) was used. All the layers were treated as Cauchy materials having the following wavelengthdependent refractive index: $n(\lambda)=A+\frac{B}{\lambda^{2}}+\frac{C}{\lambda^{4}}$, where $A, B$, and $C$ are the fitting coefficients and $\lambda$ is the wavelength. To obtain the $A, B$, and $C$ coefficients for each layer, ellipsometric measurements were performed after each modification step and fitted with the CompleteEASE software.

\section{RESULTS AND DISCUSSION}

A set of interdigitated gold electrodes as source and drain electrodes, connected via a very narrow channel $(10 \mu \mathrm{m})$ of $\mathrm{rGO}$, was used for the preparation of the transistors (Figure 1a). The interdigitated arrangement together with the liquid gate configuration leads to FETs with an extremely high transconductance and a charge carrier mobility of 30 and 15 $\mathrm{cm}^{2} \mathrm{~V}^{-1} \mathrm{~s}^{-1}$ for holes and electrons, respectively, as described in previous works. ${ }^{16,42}$ Since the covalent attachment of 


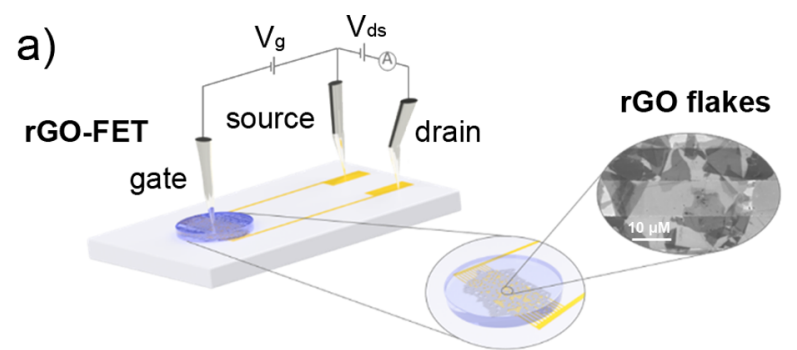

b)

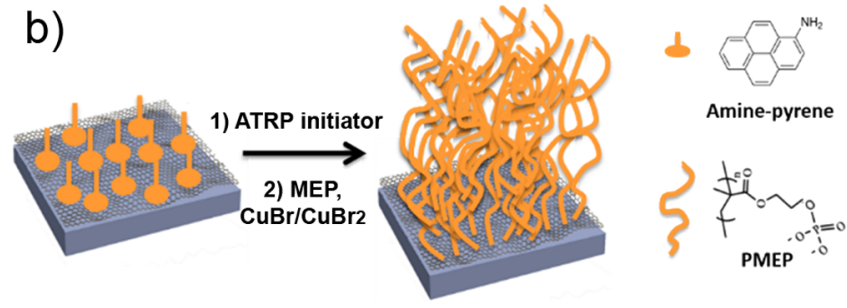

c)

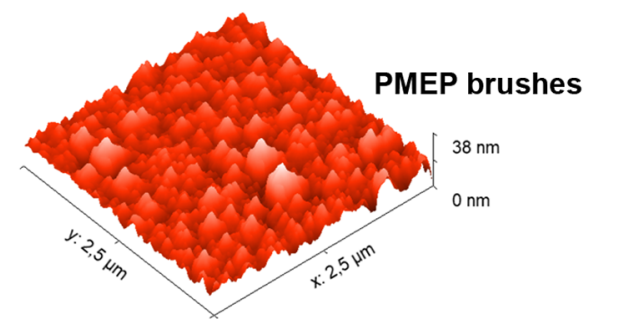

Figure 1. (a) Illustration of the liquid-gated rGO FET, the interdigitated source and drain electrodes, and SEM image of the rGO-functionalized channel. (b) Scheme of rGO FET functionalization with PMEP brushes by atom transfer radical polymerization (ATRP). (c) Topography image of PMEP brushes obtained by atomic force microscopy (AFM).

molecules on graphene disrupts the $\pi$-conjugation structure, ${ }^{45-47}$ a polymerization method that does not alter the semiconducting properties of graphene needs to be chosen. It has been shown that the self-assembly of aromatic compounds on graphene surfaces via $\pi-\pi$ stacking has no deleterious effects on the exceptional electronic quality of this material. ${ }^{48}$ With this in mind, we propose an SI-ATRP approach initiated from an amine-pyrene linker previously assembled on the $\mathrm{rGO}$ surface (Figure 1b).

The surface functionalization was confirmed using X-ray photoelectron spectroscopy (XPS). The first two spectra in Figure 2a show the successful reduction of GO to rGO using hydrazine as the intensity of the $\mathrm{C}-\mathrm{O}$ peak decreases in the presence of signals corresponding to other functional groups on the rGO surface. After the PMEP modification, the signal corresponding to $\mathrm{O}-\mathrm{C}=\mathrm{O}$ groups increased in intensity, which is consistent with the additional ester groups of PMEP. Further, the effective functionalization of the rGO surface by PMEP is evidenced by the presence of $\mathrm{P} 2 \mathrm{~s}$ and $\mathrm{P} 2 \mathrm{p}$ XPS signals (Figure $2 \mathrm{~b}$ ). In another experiment, $\mathrm{Ca}$ and $\mathrm{P}$ spectra were recorded after incubation of a PMEP-modified rGO in 1 $\mathrm{mM} \mathrm{CaCl} 2$ and subsequent rinsing with deionized water to remove the salt excess. The resulting $\mathrm{Ca} / \mathrm{P}$ ratio was determined to be 0.4 (Figure 2c) instead of 1 . We attribute this observation to two plausible effects: (i) the presence of counterion gradients within the film or (ii) the coexistence of mono- and dibasic phosphate species, instead of the sole presence of dibasic species in the brush layer. Surface modification was also proven by contact angle measurements (see Figure S1 in the Supporting Information). Further, the
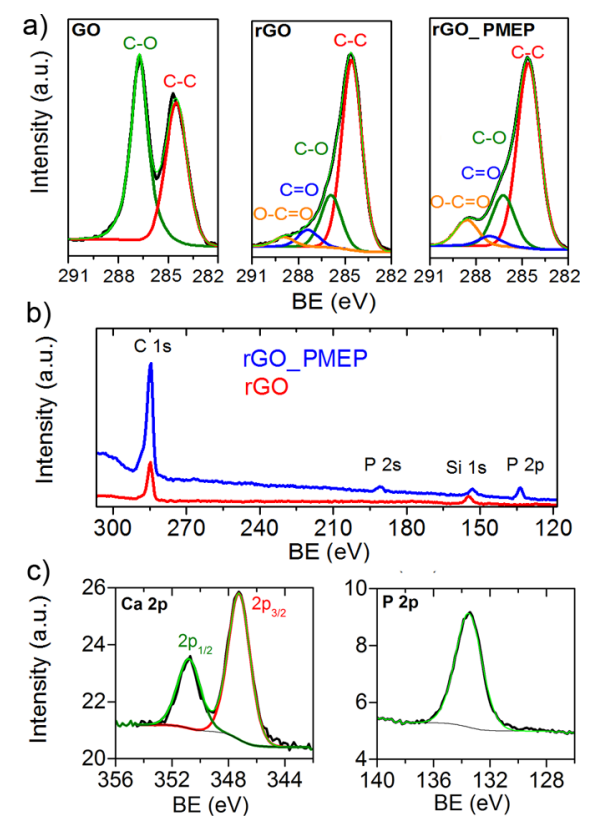

Figure 2. (a) XPS spectra in the $\mathrm{C} 1 \mathrm{~s}$ binding energy region for graphene oxide (GO), reduced graphene oxide (rGO), and rGO functionalized with PMEP brushes (rGO_PMEP). (b) Comparison of the survey XPS spectra of rGO (red) and PMEP-functionalized rGO (blue). (c) Ca $2 p$ (left) and P $2 p$ (right) energy regions of rGO_PMEP after incubation in $\mathrm{Ca}^{2+}$.

PMEP thickness was studied by ellipsometry (Figure S2), showing a film thickness of $19 \pm 3 \mathrm{~nm}$. This value is in agreement with the thickness of PMEP brushes polymerized on gold and silicon wafers using also the ATRP approach. ${ }^{44}$ The AFM topography image of an rGO substrate modified with PMEP (Figure 1c) reveals a continuous and uniform film with a roughness (in terms of the root mean square height) of $3.3 \mathrm{~nm}$ (additional scanning electron microscopy characterization is included in Supporting Information, Figure S3).

To assess the chemoresponsive properties of the PMEP brushes, the contact angle was measured with solutions containing monovalent $\left(\mathrm{K}^{+}\right)$or divalent cations $\left(\mathrm{Ca}^{2+}\right)$. Figure 3a shows contact angles of PMEP brushes prior to and after coordination with $\mathrm{Ca}^{2+}$. A marked increase in hydrophobicity was observed in the presence of the divalent cation, which is a result of a surface charge density decrease due to the PMEP coordination with $\mathrm{Ca}^{2+}$ ions. ${ }^{38}$

Sweeping the gate potential $\left(V_{\mathrm{g}}\right)$ while monitoring the current between the drain and the source $\left(I_{\mathrm{ds}}\right)$ results in transfer characteristics with a minimum conductance, corresponding to the minimum concentration of charge carriers (the so-called Dirac point). The left and right branches from this charge neutrality point represent the hole and electron conductivity regimes, respectively. ${ }^{49}$ We should note that after the MEP polymerization, a slight decrease in the FET transconductance was observed (Figure S4), which could be attributed to desorption of the rGO sheets not so strongly attached to the substrate surface. The negative charge of the PMEP brushes induces an electric field that generates positive charges in the graphene channel (i.e., holes into the valence band) and shifts the Fermi level below the Dirac point (Figure $3 b)$. When $\mathrm{Ca}^{2+}$ ions bind to the phosphate groups in the polymer brushes, the surface charge is neutralized, and the transfer characteristics shift to more negative gate values. This 
a)
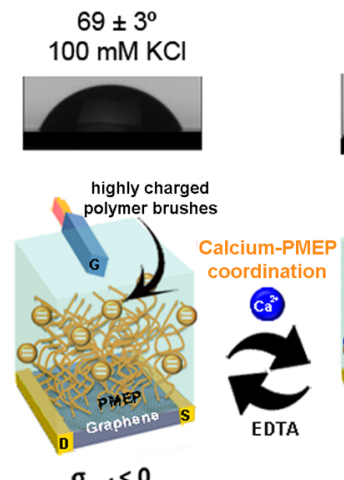

$\sigma_{\text {surf }}<0$

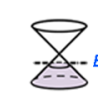
Fine-tuning of
the Dirac point
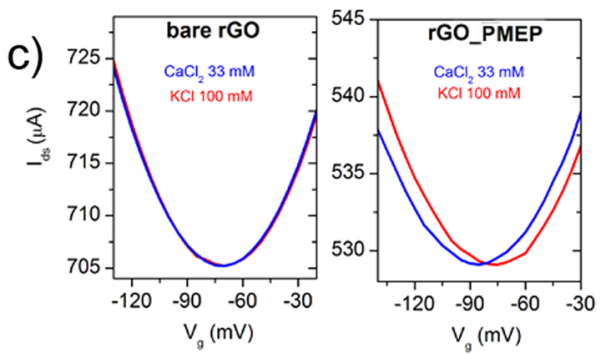

Figure 3. (a) Contact angle of rGO surfaces modified with PMEP brushes and measured with solutions containing monovalent and divalent cations: $100 \mathrm{mM} \mathrm{KCl}$ (left) and $33 \mathrm{mM} \mathrm{CaCl}_{2}$ (right). The values of the salt concentrations were chosen to keep the electrostatic screening length constant. (b) Scheme of the PMEP brushes and the Fermi level before and after the binding of $\mathrm{Ca}^{2+}$ ions. (c) Transfer characteristics for bare rGO FET (left) and rGO FET functionalized with PMEP (right) in the presence of $100 \mathrm{mM} \mathrm{KCl}$ (red) and $33 \mathrm{mM}$ $\mathrm{CaCl}_{2}$ (blue).

phenomenon can be observed by comparing the transfer characteristics in the presence and in the absence of $\mathrm{Ca}^{+2}$ (Figure 3c). For the PMEP-modified FET, the Dirac point $\left(V_{\mathrm{i}}\right)$ clearly shifts to more negative values upon binding of the divalent cations (right), whereas no signal is observed in the control measurement using nonmodified rGO FETs (left).

Figure $4 \mathrm{a}$ shows that the binding of the analyte to the polymer brushes can be reversed by complexing the $\mathrm{Ca}^{2+}$ ions using $10 \mathrm{mM}$ EDTA for $20 \mathrm{~min}$. This treatment regenerates the negative charge of the brush and shifts the Dirac point (left) and the contact angle (right) back to the dissociated PMEP values. On the other hand, in the case of $\mathrm{Ca}^{2+}$ binding, we have observed that the signal reaches a stable value after 1 min exposure. Measuring the Dirac point shift $\left(\Delta V_{\mathrm{i}}\right)$ at different concentrations of $\mathrm{Ca}^{2+}$ gives a response curve with a Langmuir-like adsorption behavior, where the polymer brush reaches a binding saturation close to $10 \mathrm{mM} \mathrm{Ca}^{+2}$ (Figure $4 \mathrm{~b}$ ). According to the experimental data taken from the linear regime, the sensitivity of the PMEP-modified FET device is 17 $\mathrm{mV}$ per decade. For the sake of comparison, current $\mathrm{Ca}^{2+}$ sensors employing more sophisticated sensing configurations exhibit sensitivities close to $25 \mathrm{mV}$ per decade. In principle, we assumed that all the monomer units are exposed to $\mathrm{Ca}^{2+}$ ions. In previous studies, it has been demonstrated that in the case of thin polymer brushes (thickness $<100 \mathrm{~nm}$ ), counterions fully penetrate the inner environment of the polymer layer. $^{50-53}$ In our experiments, the thickness of the polymer
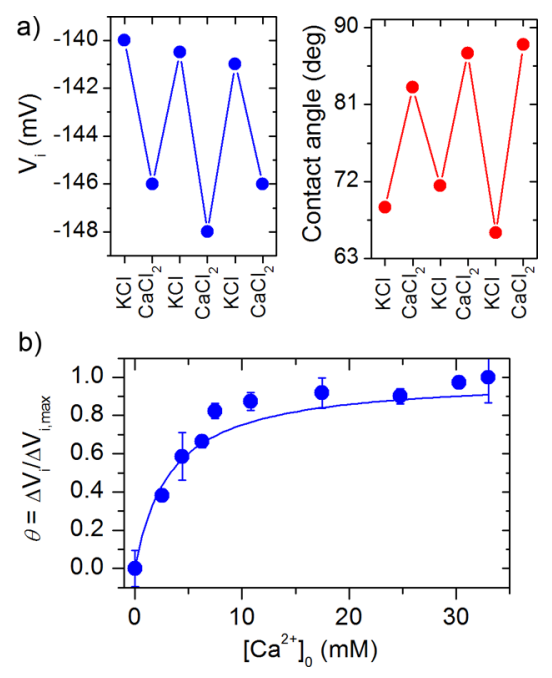

Figure 4. (a) Reversibility of the Dirac point (left) and contact angle (right) in the presence and absence of $\mathrm{Ca}^{2+}$. Between consecutive measurements, the substrates were incubated in EDTA. (b) Langmuir-Freundlich binding isotherm showing the surface coverage of PMEP coordinated by $\mathrm{Ca}^{2+}$ ions. The circles are the experimental results, and the solid line is the fitting using the model described in the text.

brushes is $\sim 20 \mathrm{~nm}$; hence, we can infer that cations penetrate through the whole thickness of the macromolecular layer.

A formalism based on the Langmuir-Freundlich binding isotherm and the Grahame equation was proposed to obtain the degree of PMEP coordination (i.e., the surface coverage) from the change in the Dirac point $\left(\Delta V_{\mathrm{i}}\right)$. The modification of the graphene surface with very thin polymer brushes facilitates the use and application of this isotherm to describe the binding process. However, we recognize that above a critical thickness value, this isotherm is no longer applicable and a more detailed analysis would be needed to describe the experiments.

The binding equilibrium of $\mathrm{Ca}^{2+}$ to the phosphate groups of the polymer brushes can be described as follows

$$
\begin{aligned}
& \left(\mathrm{RO}-\mathrm{PO}_{3}{ }^{2-}\right)+\mathrm{Ca}^{2+}=\left(\mathrm{RO}-\mathrm{PO}_{3} \mathrm{Ca}\right), K_{1} \\
& \left(\mathrm{RO}-\mathrm{HPO}_{3}{ }^{-}\right)+\mathrm{Ca}^{2+}=\left(\mathrm{RO}-\mathrm{HPO}_{3} \mathrm{Ca}^{+}\right), K_{2} \\
& \left(\mathrm{RO}-\mathrm{H}_{2} \mathrm{PO}_{3}\right)+\mathrm{Ca}^{2+}=\left(\mathrm{RO}-\mathrm{H}_{2} \mathrm{PO}_{3} \mathrm{Ca}^{2+}\right), K_{3}
\end{aligned}
$$

where parentheses are used to designate surface species and $K_{1}$, $K_{2}$, and $K_{3}$ represent the respective equilibrium constants, which are described in the Supporting Information. As we worked at $\mathrm{pH} 10,{ }^{54}\left[\left(\mathrm{RO}-\mathrm{HPO}_{3}{ }^{-1}\right)\right] \approx\left[\left(\mathrm{RO}-\mathrm{H}_{2} \mathrm{PO}_{3}\right)\right] \approx 0$, and it is possible to assume that the divalent cation coordination is described by the $K_{1}$ equilibrium. Therefore, the PMEP-Ca surface coverage expression $(\theta)$ simplifies to

$$
\theta=\frac{\left(K_{1}\left[\mathrm{Ca}^{2+}\right]_{0}\right)^{m}}{1+\left(K_{1}\left[\mathrm{Ca}^{2+}\right]_{0}\right)^{m}}
$$

where $\left[\mathrm{Ca}^{2+}\right]_{0}$ is the concentration of $\mathrm{Ca}^{2+}$ close to the PMEP brush surface (see the Supporting Information for $\left[\mathrm{Ca}^{2+}\right]_{0}$ calculation) and $m$ is called the heterogeneity index. This adsorption model takes into account the heterogeneity of the adsorption energy of the binding sites and the lateral ligand interactions where $m$ can take values $\leq 1$. $^{55}$ 
The surface charge density $(\sigma)$ is a function of the concentration of phosphate charged groups and can be expressed as follows:

$$
\sigma=2 \mathrm{e}^{-}\left[\mathrm{RO}-\mathrm{PO}_{3}^{2-}\right]
$$

As phosphate groups are neutralized by the coordination to $\mathrm{Ca}^{2+}$, the surface coverage can be written in terms of the surface charge density: $\theta=\left(\sigma_{i}-\sigma\right) / \sigma_{i}$, where $\sigma_{i}$ is the surface charge density of completely dissociated PMEP (i.e., in the absence of $\mathrm{Ca}^{2+}$ ).

On the other hand, the Grahame equation relates the surface charge density to the electrostatic potential at the interface of the polymer brush $\left(\psi_{\mathrm{s}}\right)$ :

$$
\sigma=\sqrt{8 \epsilon \epsilon_{0} k T n_{0}} \sinh \left(\frac{e \psi_{s}}{2 k T}\right)
$$

The relation can be simplified for low $\psi_{\mathrm{s}}$ to $\sigma=\epsilon \epsilon_{0} \psi_{\mathrm{s}} / \lambda_{\mathrm{D}}$ where $\lambda_{\mathrm{D}}$ is the Debye length. As the Dirac point $\left(V_{\mathrm{i}}\right)$ is proportional to the electrostatic potential, ${ }^{12}$ the surface coverage can finally be expressed as follows

$$
\theta=\Delta V_{i} / \Delta V_{i, \max }
$$

where $\Delta V_{i, \max }$ is the change of the Dirac point obtained upon saturation of $\mathrm{Ca}^{2+}$.

The dependence of the surface coverage (calculated using eq 4 ) as a function of $\left[\mathrm{Ca}^{2+}\right]_{0}$ was fitted with eq 1 to determine $K_{1}$ and $m$. Values corresponding to $K_{1}=301 \pm 42 \mathrm{M}^{-1}$ and $m=1$ \pm 0.2 were obtained. These values are consistent with previously reported association constants of calcium with other $\left(\mathrm{RO}-\mathrm{PO}_{3}{ }^{2-}\right)$ molecules such as phosphatidylinositol $\left(10 \mathrm{M}^{-1}\right.$ ) and phosphatidylinositol 4,5-bisphosphate (500 $\left.\mathrm{M}^{-1}\right) .{ }^{56}$ Moreover, the value obtained for $m$ evidences a very low degree of heterogeneity of the binding sites.

It was also observed that the transconductance of the PMEP-modified rGO FET diminishes when $\mathrm{Ca}^{2+}$ binds to the polymer brush with respect to measurements in $\mathrm{KCl}$ (Figure S5). This effect may be attributed to the collapse of the polymer brush upon binding of the divalent cation. ${ }^{4,38} \mathrm{~A}$ similar effect was observed for $\mathrm{Mg}^{2+}$. The maximum Dirac point shift upon binding of $\mathrm{Mg}^{2+}$ ions was found to be $-2 \mathrm{mV}$ (see the Supporting Information for more details). This lower shift compared to the measurements performed with $\mathrm{Ca}^{2+}$ is expected since the affinity between phosphate and calcium is higher than that corresponding to phosphate and magnesium. $^{56,57}$

\section{CONCLUSIONS}

In summary, an ion-responsive negatively charged polymer brush was grown on the graphene surface via atom transfer radical polymerization (ATRP) of poly[2-(methacryloyloxy)ethyl] phosphate (PMEP). The resulting chemoresponsive graphene-based transistor responds to the presence of the divalent ions $\mathrm{Ca}^{2+}$ and $\mathrm{Mg}^{2+}$. Our results show a reversible modulation of the Dirac point voltage (by electrostatic gating effects) due to the divalent PMEP-cation coordination. The binding of $\mathrm{Ca}^{2+}$ showed a Langmuir-type binding behavior with an affinity constant of $301 \mathrm{M}^{-1}$. This functionalization approach, initiated from amine-pyrene linkers noncovalently attached to the rGO surface, represents a powerful tool to combine functional polymer brushes and rGO-based FETs to develop chemoresponsive devices with responsive interfacial architectures. Since our polymerization approach could be extended to a wide variety of stimuli-responsive monomers, we believe that these results will have profound implications for the development of advanced sensors and smart logic gates.

\section{ASSOCIATED CONTENT}

\section{Supporting Information}

The Supporting Information is available free of charge on the ACS Publications website at DOI: 10.1021/acs.langmuir.9b00910.

Scanning electron microscopy (SEM) images of PMEPmodified rGO FETs, ellipsometric and transconductance measurements, and further PMEP characterization by contact angle measurement (PDF)

\section{AUTHOR INFORMATION}

\section{Corresponding Author}

*E-mail: azzaroni@inifta.unlp.edu.ar.

ORCID

Omar Azzaroni: 0000-0002-5098-0612

\section{Notes}

The authors declare no competing financial interest.

\section{ACKNOWLEDGMENTS}

This work was supported by CONICET, ANPCyT (PICT2016-1680, PICT-2017-1523), CEST-Competence Center for Electrochemical Surface Technologies (CEST-UNLP Partner Lab for Bioelectronics), Universidad Nacional de La Plata (PPID-X016), the Austrian Federal Ministry for Transportation, Innovation and Technology (GZ BMVIT612.166/0001-III/I1/2010), by the FFG within the comet program, and from the governments of Lower and Upper Austria. E.P. acknowledges CONICET for a scholarship. J.M.G. and O.A. are staff researchers of CONICET.

\section{REFERENCES}

(1) Novoselov, K. S.; Geim, A. K.; Morozov, S. V.; Jiang, D.; Zhang, Y.; Dubonos, S. V.; Grigorieva, I. V.; Firsov, A. A. Electric Field Effect in Atomically Thin Carbon Films. Science 2004, 306, 666-669.

(2) Novoselov, K. S.; Geim, A. K.; Morozov, S. V.; Jiang, D.; Katsnelson, M. I.; Grigorieva, I. V.; Dubonos, S. V.; Firsov, A. A. TwoDimensional Gas of Massless Dirac Fermions in Graphene. Nature 2005, 438, 197-200.

(3) Rocha, C. G.; Rümmeli, M. H.; Ibrahim, I.; Sevincli, H.; Börrnert, F.; Kunstmann, J.; Bachmatiuk, A.; Pötschke, M.; Li, W.; Makharza, S. A. M.; et al. Nanomaterials and Their Applications. In Graphene: Synthesis and Applications; Choi, W., Lee, J., Eds.; CRC Press: 2012; pp 1-24.

(4) Liu, S.; Jamali, S.; Liu, Q.; Maia, J.; Baek, J.-B.; Jiang, N.; Xu, M.; Dai, L. Conformational Transitions of Polymer Brushes for Reversibly Switching Graphene Transistors. Macromolecules 2016, 49, 74347441.

(5) Gobbi, M.; Bonacchi, S.; Lian, J. X.; Vercouter, A.; Bertolazzi, S.; Zyska, B.; Timpel, M.; Tatti, R.; Olivier, Y.; Hecht, S.; Nardi, M. V.; Beljonne, D.; Orgiu, E.; et al. Collective Molecular Switching in Hybrid Superlattices for Light-Modulated Two-Dimensional Electronics. Nat. Commun. 2018, 9, 2661.

(6) Mao, S.; Chang, J.; Pu, H.; Lu, G.; He, Q.; Zhang, H.; Chen, J. Two-Dimensional Nanomaterial-Based Field-Effect Transistors for Chemical and Biological Sensing. Chem. Soc. Rev. 2017, 46, 68726904.

(7) Kim, M.; Safron, N. S.; Huang, C.; Arnold, M. S.; Gopalan, P. Light-Driven Reversible Modulation of Doping in Graphene. Nano Lett. 2012, 12, 182-187. 
(8) Xu, Y.; Sun, H.; Liu, A.; Zhu, H.-H.; Li, W.; Lin, Y.-F.; Noh, Y.Y. Doping: A Key Enabler for Organic Transistors. Adv. Mater. 2018, 30, 13-19.

(9) Lee, H.; Paeng, K.; Kim, I. S. A Review of Doping Modulation in Graphene. Synth. Met. 2018, 244, 36-47.

(10) Fu, W.; Jiang, L.; van Geest, E. P.; Lima, L. M. C.; Schneider, G. F. Sensing at the Surface of Graphene Field-Effect Transistors. Adv. Mater. 2017, 29, 1603610.

(11) Yan, F.; Zhang, M.; Li, J. Solution-Gated Graphene Transistors for Chemical and Biological Sensors. Adv. Healthcare Mater. 2014, 3, 313-331.

(12) Wang, Y. Y.; Burke, P. J. Polyelectrolyte Multilayer Electrostatic Gating of Graphene Field-Effect Transistors. Nano Res. 2014, 7, $1650-1658$.

(13) Piccinini, E.; Alberti, S.; Longo, G. S.; Berninger, T.; Breu, J.; Dostalek, J.; Azzaroni, O.; Knoll, W. Pushing the Boundaries of Interfacial Sensitivity in Graphene FET Sensors: Polyelectrolyte Multilayers Strongly Increase the Debye Screening Length. J. Phys. Chem. C 2018, 122, 10181-10188.

(14) Lerner, M. B.; Matsunaga, F.; Han, G. H.; Hong, S. J.; Xi, J.; Crook, A.; Perez-Aguilar, J. M.; Park, Y. W.; Saven, J. G.; Liu, R.; et al. Scalable Production of Highly Sensitive Nanosensors Based on Graphene Functionalized with a Designed G Protein-Coupled Receptor. Nano Lett. 2014, 14, 2709-2714.

(15) Kim, D.-J.; Sohn, Y. I.; Jung, J.-H.; Yoon, O. J.; Lee, N.-E.; Park, J.-S. Reduced Graphene Oxide Field-Effect Transistor for Label-Free Femtomolar Protein Detection. Biosens. Bioelectron. 2013, 41, 621626.

(16) Piccinini, E.; Bliem, C.; Reiner-Rozman, C.; Battaglini, F.; Azzaroni, O.; Knoll, W. Enzyme-Polyelectrolyte Multilayer Assemblies on Reduced Graphene Oxide Field-Effect Transistors for Biosensing Applications. Biosens. Bioelectron. 2017, 92, 661-667.

(17) Ohno, Y.; Maehashi, K.; Matsumoto, K. Label-Free Biosensors Based on Aptamer-Modified Graphene Field-Effect Transistors. J. Am. Chem. Soc. 2010, 132, 18012-18013.

(18) Dill, K. A.; Shortle, D. Denatured States of Proteins. Annu. Rev. Biochem. 1991, 60, 795-825.

(19) Iskierko, Z.; Noworyta, K.; Sharma, P. S. Molecular Recognition by Synthetic Receptors: Application in Field-Effect Transistor Based Chemosensing. Biosens. Bioelectron. 2018, 109, 5062.

(20) Irigoyen, J.; Moya, S. E.; Iturri, J. J.; Llarena, I.; Azzaroni, O.; Donath, E. Specific $\zeta$-Potential Response of Layer-by-Layer Coated Colloidal Particles Triggered by Polyelectrolyte Ion Interactions. Langmuir 2009, 25, 3374-3380.

(21) Azzaroni, O.; Moya, S.; Farhan, T.; Brown, A. A.; Huck, W. T. S. Switching the Properties of Polyelectrolyte Brushes via "Hydrophobic Collapse.”. Macromolecules 2005, 38, 10192-10199.

(22) Azzaroni, O.; Szleifer, I. Polymer and Biopolymer Brushes: For Materials Science and Biotechnology; Azzaroni, O., Szleifer, I., Eds.; John Wiley \& Sons, Inc.: Hoboken, NJ, USA, 2017.

(23) Giussi, J. M.; Cortez, M. L.; Marmisollé, W. A.; Azzaroni, O. Practical Use of Polymer Brushes in Sustainable Energy Applications: Interfacial Nanoarchitectonics for High-Efficiency Devices. Chem. Soc. Rev. 2019, 48, 814-849.

(24) Azzaroni, O.; Moya, S. E.; Brown, A. A.; Zheng, Z.; Donath, E.; Huck, W. T. S. Polyelectrolyte Brushes as Ink Nanoreservoirs for Microcontact Printing of Ionic Species with Poly(dimethyl Siloxane) Stamps. Adv. Funct. Mater. 2006, 16, 1037-1042.

(25) Yameen, B.; Kaltbeitzel, A.; Langer, A.; Müller, F.; Gösele, U.; Knoll, W.; Azzaroni, O. Highly Proton-Conducting Self-Humidifying Microchannels Generated by Copolymer Brushes on a Scaffold. Angew. Chem. Int. Ed. 2009, 48, 3124-3128.

(26) Brown, A. A.; Azzaroni, O.; Fidalgo, L. M.; Huck, W. T. S. Polymer Brush Resist for Responsive Wettability. Soft Matter 2009, 5, 2738.

(27) Brunsen, A.; Cui, J.; Ceolín, M.; del Campo, A.; Soler-Illia, G. J. A. A.; Azzaroni, O. Light-Activated Gating and Permselectivity in
Interfacial Architectures Combining "caged" Polymer Brushes and Mesoporous Thin Films. Chem. Commun. 2012, 48, 1422-1424.

(28) Cui, J.; Azzaroni, O.; del Campo, A. Polymer Brushes with Phototriggered and Phototunable Swelling and $\mathrm{pH}$ Response. Macromol. Rapid Commun. 2011, 32, 1699-1703.

(29) Brown, A. A.; Azzaroni, O.; Huck, W. T. S. Photoresponsive Polymer Brushes for Hydrophilic Patterning. Langmuir 2009, 25, $1744-1749$.

(30) Cui, J.; Nguyen, T.-H.; Ceolín, M.; Berger, R.; Azzaroni, O.; del Campo, A. Phototunable Response in Caged Polymer Brushes. Macromolecules 2012, 45, 3213-3220.

(31) Yameen, B.; Ali, M.; Álvarez, M.; Neumann, R.; Ensinger, W.; Knoll, W.; Azzaroni, O. A Facile Route for the Preparation of AzideTerminated Polymers. "Clicking" Polyelectrolyte Brushes on Planar Surfaces and Nanochannels. Polym. Chem. 2010, 1, 183-192.

(32) Matsumoto, A.; Sato, N.; Sakata, T.; Yoshida, R.; Kataoka, K.; Miyahara, Y. Chemical-to-Electrical-Signal Transduction Synchronized with Smart Gel Volume Phase Transition. Adv. Mater. 2009, 21, $4372-4378$

(33) Guo, X.; Liu, J.; Liu, F.; She, F.; Zheng, Q.; Tang, H.; Ma, M.; Yao, S. Label-Free and Sensitive Sialic Acid Biosensor Based on Organic Electrochemical Transistors. Sensors Actuators, B Chem. 2017, 240, 1075-1082.

(34) Fillaud, L.; Petenzi, T.; Pallu, J.; Piro, B.; Mattana, G.; Noel, V. Switchable Hydrogel-Gated Organic Field-Effect Transistors. Langmuir 2018, 34, 3686-3693.

(35) Hess, L. H.; Lyuleeva, A.; Blaschke, B. M.; Sachsenhauser, M.; Seifert, M.; Garrido, J. A.; Deubel, F. Graphene Transistors with Multifunctional Polymer Brushes for Biosensing Applications. ACS Appl. Mater. Interfaces 2014, 6, 9705-9710.

(36) Heller, I.; Chatoor, S.; Männik, J.; Zevenbergen, M. A. G.; Dekker, C.; Lemay, S. G. Influence of Electrolyte Composition on Liquid-Gated Carbon Nanotube and Graphene Transistors. J. Am. Chem. Soc. 2010, 132, 17149-17156.

(37) Brunsen, A.; Díaz, C.; Pietrasanta, L. I.; Yameen, B.; Ceolín, M.; Soler-Illia, G. J. A. A.; Azzaroni, O. Proton and Calcium-Gated Ionic Mesochannels: Phosphate-Bearing Polymer Brushes Hosted in Mesoporous Thin Films as Biomimetic Interfacial Architectures. Langmuir 2012, 28, 3583-3592.

(38) Liu, Z.; Liu, J.; Hu, H.; Yu, B.; Chen, M.; Zhou, F. Reversible Hydration and Dehydration of Polyanionic Brushes Bearing Carboxylate, Phosphate and Sulfonate Side Groups: A Comparative AFM Study. Phys. Chem. Chem. Phys. 2008, 10, 7180-7185.

(39) Zoppe, J. O.; Ataman, N. C.; Mocny, P.; Wang, J.; Moraes, J.; Klok, H. A. Surface-Initiated Controlled Radical Polymerization: State-of-the-Art, Opportunities, and Challenges in Surface and Interface Engineering with Polymer Brushes. Chem. Rev. 2017, 117, $1105-1318$

(40) Xie, G.; Khabibullin, A.; Pietrasik, J.; Yan, J.; Matyjaszewski, K. Polymer Brushes by Atom Transfer Radical Polymerization. In Polymer and Biopolymer Brushes; Azzaroni, O., Szleifer, I., Eds.; John Wiley \& Sons, Inc.: Hoboken, NJ, USA, 2017; pp 29-95.

(41) Azzaroni, O. Polymer Brushes Here, There, and Everywhere: Recent Advances in Their Practical Applications and Emerging Opportunities in Multiple Research Fields. J. Polym. Sci. Part A Polym. Chem. 2012, 50, 3225-3258.

(42) Bliem, C.; Piccinini, E.; Knoll, W.; Azzaroni, O. Enzyme Multilayers on Graphene-Based FETs for Biosensing Applications. In Enzyme Nanoarchitectures: Enzymes Armored with Graphene; Kumar, C. V., Ed.; Elsevier: 2018; pp 23-46, DOI: 10.1016/ bs.mie.2018.06.001.

(43) Fenoy, G. E.; Giussi, J. M.; von Bilderling, C.; Maza, E. M.; Pietrasanta, L. I.; Knoll, W.; Marmisollé, W. A.; Azzaroni, O. Reversible Modulation of the Redox Activity in Conducting Polymer Nanofilms Induced by Hydrophobic Collapse of a Surface-Grafted Polyelectrolyte. J. Colloid Interface Sci. 2018, 518, 92-101.

(44) Zhou, F.; Huck, W. T. S. Three-Stage Switching of Surface Wetting Using Phosphate-Bearing Polymer Brushes. Chem. Commun. 2005, 5999-6001. 
(45) Loh, K. P.; Bao, Q.; Ang, P. K.; Yang, J. The Chemistry of Graphene. J. Mater. Chem. 2010, 20, 2277-2289.

(46) Niyogi, S.; Bekyarova, E.; Itkis, M. E.; Zhang, H.; Shepperd, K.; Hicks, J.; Sprinkle, M.; Berger, C.; Lau, C. N.; Deheer, W. A.; et al. Spectroscopy of Covalently Functionalized Graphene. Nano Lett. 2010, 10, 4061-4066.

(47) Ramasubramaniam, A.; Selhorst, R.; Alon, H.; Barnes, M. D.; Emrick, T.; Naveh, D. Combining 2D Inorganic Semiconductors and Organic Polymers at the Frontier of the Hard-soft Materials Interface. J. Mater. Chem. C 2017, 5, 11158-11164.

(48) Fu, W.; Nef, C.; Tarasov, A.; Wipf, M.; Stoop, R.; Knopfmacher, O.; Weiss, M.; Calame, M.; Schönenberger, C. High Mobility Graphene Ion-Sensitive Field-Effect Transistors by Noncovalent Functionalization. Nanoscale 2013, 5, 12104.

(49) Reiner-Rozman, C.; Larisika, M.; Nowak, C.; Knoll, W. Graphene-Based Liquid-Gated Field Effect Transistor for Biosensing: Theory and Experiments. Biosens. Bioelectron. 2015, 70, 21-27.

(50) Moya, S. E.; Azzaroni, O.; Kelby, T.; Donath, E.; Huck, W. T. S. Explanation for the Apparent Absence of Collapse of Polyelectrolyte Brushes in the Presence of Bulky Ions. J. Phys. Chem. B 2007, 111, 7034-7040.

(51) Rodríguez Presa, M. J.; Gassa, L. M.; Azzaroni, O.; Gervasi, C. A. Estimating Diffusion Coefficients of Probe Molecules into Polyelectrolyte Brushes by Electrochemical Impedance Spectroscopy. Anal. Chem. 2009, 81, 7936-7943.

(52) Choi, E.-Y.; Azzaroni, O.; Cheng, N.; Zhou, F.; Kelby, T.; Huck, W. T. S. Electrochemical Characteristics of Polyelectrolyte Brushes with Electroactive Counterions. Langmuir 2007, 23, 1038910394.

(53) García, T. A.; Gervasi, C. A.; Rodríguez Presa, M. J.; Otamendi, J. I.; Moya, S. E.; Azzaroni, O. Molecular Transport in Thin Thermoresponsive Poly( N -Isopropylacrylamide) Brushes with Varying Grafting Density. J. Phys. Chem. C 2012, 116, 13944-13953.

(54) Yameen, B.; Ali, M.; Neumann, R.; Ensinger, W.; Knoll, W.; Azzaroni, O. Proton-Regulated Rectified Ionic Transport through Solid-State Conical Nanopores Modified with Phosphate-Bearing Polymer Brushes. Chem. Commun. 2010, 46, 1908-1910.

(55) Laucirica, G.; Marmisollé, W. A.; Azzaroni, O. Dangerous liaisons: Anion-Induced Protonation in Phosphate-Polyamine Interactions and Their Implications for the Charge States of Biologically Relevant Surfaces. Phys. Chem. Chem. Phys. 2017, 19, 8612-8620.

(56) Toner, M.; Vaio, G.; McLaughlin, A.; McLaughlin, S. Adsorption of Cations to Phosphatidylinositol 4,5-Bisphosphate. Biochemistry 1988, 27, 7435-7443 10.1021/bi00419a039. .

(57) Bi, X.; Wong, W. L.; Ji, W.; Agarwal, A.; Balasubramanian, N.; Yang, K. L. Development of Electrochemical Calcium Sensors by Using Silicon Nanowires Modified with Phosphotyrosine. Biosens. Bioelectron. 2008, 23, 1442-1448. 